\title{
Exponential suppression of interlayer conductivity in very anisotropic quasi-two-dimensional compounds in high magnetic field
}

\author{
P. D. Grigoriev \\ L. D. Landau Institute for Theoretical Physics, Chernogolovka, 142432, Russi踏
}

(Dated: October 21, 2018)

\begin{abstract}
It is shown that in rather strong magnetic field the interlayer electron conductivity is exponentially damped by the Coulomb barrier arising from the formation of polaron around each localized electron state. The theoretical model is developed to describe this effect, and the calculation of the temperature and field dependence of interlayer magnetoresistance is performed. The results obtained agree well with the experimental data in GaAs/AlGaAs heterostructures and in strongly anisotropic organic metals. The proposed theory allows to use the experiments on interlayer magnetoresistance to investigate the electron states, localized by magnetic field and disorder.
\end{abstract}

PACS numbers: 72.15.Gd,73.43.Qt,74.70.Kn,74.72.-h

\section{INTRODUCTION}

Layered materials is a wide class of compounds of very strong scientific and technological impact. These materials are composed of a stack of two-dimensional (2D) conducting layers with weak interlayer electron coupling, determined by the interlayer transfer integral $t_{z}$. Such layered structure may be of natural molecular origin, as, e.g., in cuprates, pnictides, organic metals or graphite, or of artificial origin, as in heterostructures or intercalated graphites. The small value of $t_{z}$, which is much smaller than the in-plane Fermi energy $E_{F}$, provides these compounds with highly anisotropic electronic properties.

Magnetoresistance is traditionally used to explore the internal electron structure of metals. [1-3] Magnetoresistance in layered compounds has many special features. These are beats of magnetic quantum oscillations (MQO), 3] angular magnetoresistance oscillations (AMRO), [4 6] slow oscillations of magnetoresistance 7, [8], the phase shift of beats of MQO, [8, 9] etc. The angular dependence of magnetoresistance is widely used to investigate the electronic structure of various layered compounds: organic metals (see, e.g., Refs. [10 13] for reviews), cuprate high-temperature superconductors, 14 17] heterostructures 18] etc. The AMRO are well understood [5, 6] in the framework of the standard semiclassical 3D theory, based on the Boltzmann transport equation, which gives the Shockley-Chambers formula [1] for the angular dependence of magnetoresistance. The recent theoretical works [14, 19, 20] on AMRO only provide the calculations based on this formula, which are useful for its practical application to extract the Fermi-surface parameters from the experimental data.

There is one important feature of the angular dependence of interlayer magnetoresistance, observed in various strongly anisotropic layered compounds, which can-

\footnotetext{
* Temporarily at Laboratoire de Physique des Solides, Universite Paris-Sud 11, 91405, Orsay, France; Electronic address: grigorev@itp.ac.ru
}

not be described in the framework of the standard 3D theory. This is the so-called coherent-incoherent transition of interlayer magnetoresistance, induced by disorder and by magnetic field component $B_{z}$ perpendicular to the conducting layers. 21 24] This transition occurs in layered compounds with extremely high quasi2D anisotropy, namely, when the interlayer transfer integral $t_{z}$ is less than the cyclotron energy $\hbar \omega_{c}=$ $\hbar e B_{z} / m^{*} c$ and than the Landau level (LL) broadening $\Gamma_{0}$ due to impurities. This coherent-incoherent transition or crossover is sometimes accompanied by the metalinsulator transition [21, 25] and is always characterized by a strong growth of interlayer magnetoresistance $R_{z z}$ with the increase of $B_{z},[21,26]$ while the component $B_{\|}$ of magnetic field parallel to the conducting layers only slightly affects the interlayer conductivity at weak magnetic field. 23, 24] It is important that $R_{z z}\left(B_{z}\right)$ grows not only in the maxima of MQO of magnetoresistance, when the chemical potential is situated in the gap between LLs, but also in the minima of magnetoresistance, when the chemical potential is exactly on the LLs and when there is no any gap of electron states at the Fermi level. The 2D formula 27] for the Shubnikov-de Haas effect, based on the standard 3D theory of interlayer magnetoresistance and applied to the extremely anisotropic quasi2D limit, does not describe this effect. Moreover, the first direct comparison between the magnetoresistance in the coherent 3D and the so-called "weakly incoherent" regimes did not reveal any considerable differences. 28] Recently it was shown, 29 31 that although the initial model in Ref. [28] is valid for the weakly incoherent limit, the calculation of the electron Green's functions in disordered 2D layer in Ref. [28] is incorrect, because the impurity scattering is incorrectly treated via a constant imaginary part of the electron self-energy similar to $3 \mathrm{D}$ case. More rigorous calculations show (see, e.g. in Ref. [32]) that in strong magnetic field, in addition to the oscillations due to LL quantization, the imaginary part of the electron self energy monotonically increases with magnetic field $\propto \sqrt{B_{z}}$. [32] This monotonic increase leads 29 31 to the similar increase of the Dingle temperature and of the monotonic part of magnetoresistance 
$\propto \sqrt{B_{z}}$, which also changes the angular dependence of magnetoresistance. This improvement [29, 30] of the theory can explain some experimental observations [33] of the monotonic growth of interlayer magnetoresistance as function of $B_{z}$ when $\Gamma_{0}, t_{z} \lesssim \hbar \omega_{c}$. However, in many compounds the much stronger than $\propto \sqrt{B_{z}}$ growth of interlayer magnetoresistance has been observed. [21 26] To explain this rapid growth of $R_{z z}\left(B_{z}\right)$, the variable-range hopping mechanism of interlayer electron transport has been proposed, 34] similar to the in-layer transport in the regime of quantum Hall effect (QHE). Although a reasonable agreement with experiment has been achieved in Ref. 34] basing on the semi-phenomenological consideration, the underlying theoretical model is still unclear. First, the actual electron interlayer hopping is not variable-range, as in Ref. [34], but always to the adjacent layer, i.e. on the interlayer distance $d$. Moreover, the AMRO still exist in this limit, which means that during the interlayer hopping the in-plane electron wave function is conserved, i.e. the interlayer tunnelling term in the Hamiltonian conserves the in-plane electron momentum. This Hamiltonian is given by Eqs. (11)-(3) below. Without electron-electron (e-e) and electron-phonon (eph) interaction it has been studied in Refs. [29 31] (see Eqs. (8)-(10) of Ref. [29]). However, the comparison with some experiments shows that the results of Refs. [29 31] are applicable only in the intermediate magnetic fields, when $\Gamma_{0}, t_{z}<\hbar \omega_{c}$, but the magnetic field is still too weak to induce the metal-insulator transition.

The aim of the present paper is to generalize the model of Ref. 29] to describe the strong increase of interlayer magnetoresistance and the metal-insulator transition in very high magnetic field.

\section{THE MODEL}

The quasi-2D layered electron system with disorder and interaction is described by the following Hamiltonian, which contains four main terms:

$$
\hat{H}=\hat{H}_{0}+\hat{H}_{t}+\hat{H}_{I}+\hat{H}_{i n t} .
$$

The first term $\hat{H}_{0}$ is the Hamiltonian of the noninteracting 2D electron gas in magnetic field summed over all layers. The second term in Eq. (11) gives the coherent electron tunnelling between two adjacent layers:

$$
\hat{H}_{t}=2 t_{z} \sum_{j} \int d^{2} \boldsymbol{r}\left[\Psi_{j}^{\dagger}(\boldsymbol{r}) \Psi_{j-1}(\boldsymbol{r})+\Psi_{j-1}^{\dagger}(\boldsymbol{r}) \Psi_{j}(\boldsymbol{r})\right]
$$

where $\Psi_{j}(\boldsymbol{r})$ and $\Psi_{j}^{\dagger}(\boldsymbol{r})$ are the creation (annihilation) operators of an electron on the layer $j$ at the point $\boldsymbol{r}$. This interlayer tunnelling Hamiltonian is called "coherent" because it conserves the in-layer coordinate dependence of the electron wave function (in other words, it conserves the in-plane electron momentum) after the in- terlayer tunnelling. The third term

$$
\hat{H}_{I}=\sum_{i} \int d^{3} \mathbf{r} V_{i}(\mathbf{r}) \Psi^{\dagger}(\boldsymbol{r}) \Psi(\boldsymbol{r})
$$

gives the electron interaction with impurity potential. The last term $\hat{H}_{\text {int }}$ describes the electron-electron and electron-phonon interaction. The presence of this term differs our model from that in Refs. [29 31]. Even without $\hat{H}_{\text {int }}$ and for the case of point-like impurity potential, the exact solution of this Hamiltonian is not achievable. After inclusion of the interaction term $\hat{H}_{\text {int }}$, we can only catch the main physical effects in this system to describe the interlayer electron transport.

In the limit, $t_{z} \ll \Gamma_{0}, \hbar \omega_{c}$, the interlayer hopping $t_{z}$ can be considered as a small perturbation for the uncoupled stack of $2 \mathrm{D}$ metallic layers. The 2D disordered electron system in magnetic field has been extensively studied in connection to the quantum Hall effect. With the increase of magnetic field the crossover from the diffusive dynamics and classical Shubnikov-de Haas effect to the in-plane localization of electron states takes place. In Ref. 35] this crossover is described in detail, and the value of the crossover field $B_{c}$ is estimated for the various types of impurity potential. Although for point-like impurity potential the crossover field $B_{c}$ was not calculated in Ref. [35], the general criteria that $B_{c}$ corresponds to field when the nearest Landau levels (LLs) become separated, i.e. when $\hbar \omega_{c} \gtrsim \Gamma_{0}$, can be used. During this crossover the localization length $\xi$ of the $2 \mathrm{D}$ electron states decreases exponentially from infinity to the value 35,44

$$
\xi \approx R_{c}
$$

where the classical cyclotron (Larmor) radius

$$
R_{c}=\hbar k_{F} c / e B_{z}=k_{F} l_{H}^{2}=2 \nu / k_{F},
$$

$k_{F}$ is the in-plane Fermi momentum, $l_{H}=\sqrt{\hbar c / e B_{z}}$ is the magnetic length, and the LL filling factor $\nu=$ $F / B_{z}$, where $F=k_{F}^{2} c \hbar / 2 e$ is the MQO frequency. In the quantum limit $\nu \leq 1$ the localization length $\xi \approx l_{H}$.

In many layered materials the in-plane electron dispersion is anisotropic, and the in-plane FS has the shape of elongated ellipse with semi-axes given by the wave vectors $k_{F 1}$ and $k_{F 2}$. Then, in the absence of impurities, the electron classical orbit is also an ellipse with the main radii

$$
R_{c 1} \approx 2 \nu / k_{F 1}, R_{c 2} \approx 2 \nu / k_{F 2} .
$$

This equation is a particular (elliptic) case of the wellknown property that in magnetic field the electron orbits in coordinate and momentum spaces differ by the 90 degrees rotation only. The in-plane anisotropy of the localization length $\xi$ is the same as the anisotropy of the cyclotron radius $R_{c}, \xi_{i} \propto R_{c i}$, because the electron diffusion along the conducting layer goes via the jumps from one cyclotron orbit to another due to impurity scattering, and the average jump distance is proportional to the 
size of the electron orbit in this direction. Hence, the localization length in two main directions is

$$
\xi_{1} \approx R_{c 1}, \xi_{2} \approx R_{c 2}
$$

Any localized electron state is covered by a polaron, which comes from the static lattice deformation and from the rearrangement of the neighboring electrons. Since the electron localization length $\xi \sim R_{c}$ is larger than the Debye screening radius, the charge density $\rho(\mathbf{r})$ induced by this polaron follows the square of the electron wave function amplitude $\left|\Psi_{e}(\mathbf{r})\right|^{2}$. Hence, this polaron creates a positive charge distributed on the area

$$
A_{0} \approx \pi \xi_{1} \xi_{2}
$$

Along the interlayer direction the induced charge also follows the electron wave function $\left|\Psi_{e}(z)\right|^{2}$ and is distributed within one crystal layer, because otherwise there would be a nonuniform charge distribution along the $z$ axis. This polaron lowers the energy of each localized electron.

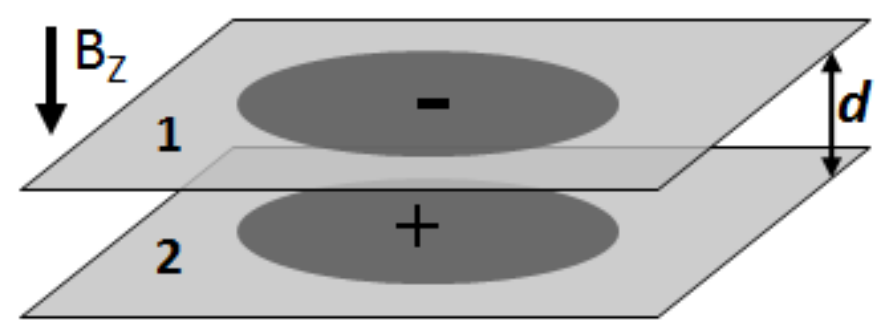

FIG. 1: The schematic view of the charge distributions (darkgray areas) of polaron (lower layer) and of the electron (upper layer) after an electron jump to the adjacent layer.

To jump to the adjacent conducting layer the electron now must overcome the Coulomb attraction of polaron, which creates the potential barrier. To estimate the value $E_{c}$ of this Coulomb barrier, consider the following electrostatic problem, schematically shown in Fig. 11. The flat capacitor has charge $e$ separated by the interlayer distance $d$. The energy stored in this capacitor is

$$
E_{c} \approx 2 \pi e^{2} d / \varepsilon A_{0},
$$

where $\varepsilon$ is the dielectric constant of the media.

\section{ESTIMATES OF INTERLAYER MAGNETORESISTANCE AT THE METAL-INSULATOR TRANSITION}

The Coulomb energy $E_{c}$, given by Eq. (9), affects the interlayer electron transport when it becomes comparable to the band-width in the interlayer direction: $E_{c} \gtrsim 4 t_{z}$. Substituting Eqs. (6)-(8) into Eq. (97), one can estimate the crossover magnetic field $B_{c}$, above which the
Coulomb energy hampers the interlayer electron transport:

$$
B_{c} \approx F \sqrt{8 \varepsilon t_{z} / e^{2} d k_{F 1} k_{F 2}}
$$

where $F=k_{F 1} k_{F 2} c \hbar / 2 e$ is the MQO frequency. Sometimes, it is convenient to use the crossover LL filling number $\nu_{c}$, which corresponds to the field in Eq. (10):

$$
\nu_{c} \approx \sqrt{e^{2} d k_{F 1} k_{F 2} / 8 \varepsilon t_{z}},
$$

At $B>B_{c}$, or at $\nu<\nu_{c}$, the interlayer electron transport becomes thermally activated. One could expect that at $B>B_{c}$

$$
\sigma_{z z}\left(B_{z}\right) / \sigma_{z z}(0) \sim \exp \left(-E_{c} / T\right) .
$$

This simple formula does not take into account two effects. First, the finite interlayer transfer integral effectively reduces the activation energy $E_{c}$ needed for an electron to jump to the next layer by the bandwidth $4 t_{z}$. This can be approximately taken into account by an effective increase of temperature in Eq. (12) according to the rule

$$
T \rightarrow T^{*} \approx T+4 t_{z}
$$

Second, the finite probability of interlayer jumping delocalizes the electrons, which increases the effective inplane electron localization length $\xi$ and reduces the Coulomb energy $E_{c}$ in Eq. (9). Third, the electron localization length vary from one state to another, and one actually has a distribution of localization length with function $P(\xi)$. This function has maximum at $\xi \sim R_{c}$ and decrease rapidly at $\xi \gg R_{c}$. However, the tails of the function $P(\xi)$, which play the crucial role for the in-plane conductivity, are also important for the interlayer transport because of the exponential dependence on $\xi$ in Eq. (12). It can be argued that the probability that the electron localization area $A$ is much larger than $A_{0} \approx \pi R_{c 1} R_{c 2}$ decreases exponentially:

$$
P(A) \sim\left(A / A_{0}\right) \exp \left(-A / A_{0}\right) .
$$

Combining Eqs. (9), (14) and (12) we obtain the suppression factor of interlayer conductivity

$$
\begin{aligned}
\frac{\sigma_{z z}\left(B_{z}\right)}{\sigma_{z z}(0)} & \sim \int_{0}^{\infty} \frac{d A}{A_{0}} \frac{A}{A_{0}} \exp \left(\frac{-A}{A_{0}}\right) \exp \left(\frac{-2 \pi e^{2} d}{\varepsilon T^{*} A}\right) \\
& =X^{2} K_{2}(X) / 2 \equiv \alpha(X),
\end{aligned}
$$

where $K_{2}(X)$ is the modified Bessel's function of the second kind, and

$$
X \equiv \sqrt{8 \pi e^{2} d / \varepsilon T^{*} A_{0}}=\sqrt{4 E_{c} / T^{*}}
$$

At $X \gg 1 \mathrm{Eq}$. (15) simplifies to

$$
\sigma_{z z}\left(B_{z}\right) / \sigma_{z z}(0) \sim \sqrt{\pi}(X / 2)^{3 / 2} \exp (-X) .
$$

In Fig. 2 we plot the inverse function $R_{z z}\left(B_{z}\right) / R_{z z}(0)=$ $\sigma_{z z}(0) / \sigma_{z z}\left(B_{z}\right) \propto \alpha^{-1}(X)$. 


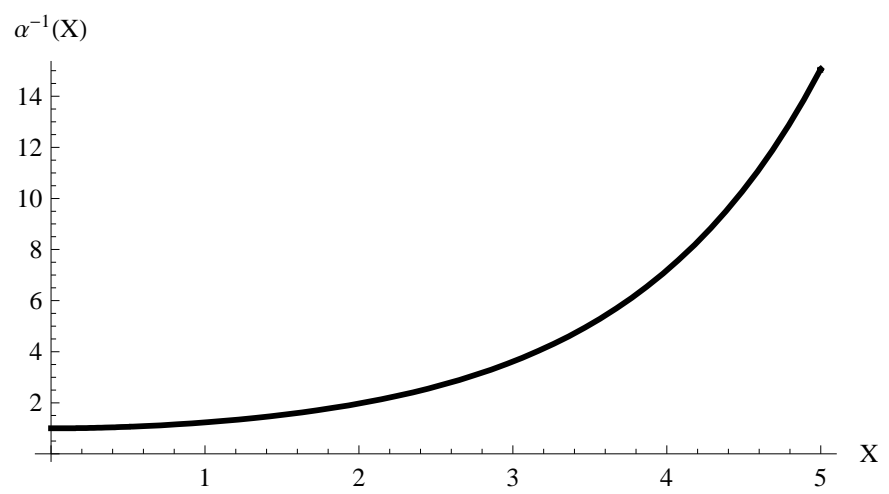

FIG. 2: The function $\alpha^{-1}(X)$ from Eq. (15). The predicted ratio $R_{z z}\left(B_{z}\right) / R_{z z}(0) \sim \alpha^{-1}(X)$ is given by the same curve, because $X \propto B_{z}$, as follows from Eqs. (16), (8) and (7).

\section{COMPARISON WITH EXPERIMENTS}

The increase of interlayer magnetoresistance described above is rather general. It has been observed in many compounds, including heterostructures [18] and various organic metals. 21 26, 45] This effect, probably, also occurs in pnictides and cuprate high-Tc superconducting materials. For comparison with experiment we choose two completely different materials: the GaAs/AlGaAs semiconductor superlattice [18] and the organic metal $\beta$ "(BEDT-TTF $)_{2} \mathrm{SF}_{5} \mathrm{CH}_{2} \mathrm{CF}_{2} \mathrm{SO}_{3}$. 25] This choice is stipulated by the small value of the interlayer transfer integral $t_{z}$ in both these materials, which makes possible the in-plane electron localization even at moderate magnetic field. In GaAs/AlGaAs superlattices the interlayer transfer integral $t_{z}$ can be controlled artificially. In the sample $\# 2$ of GaAs/AlGaAs in Ref. [18] $t_{z}=0.03 \mathrm{meV}=0.35 \mathrm{~K}$ and the interlayer distance $d \approx 300 \AA$. In $\beta "$ "-(BEDT$\mathrm{TTF})_{2} \mathrm{SF}_{5} \mathrm{CH}_{2} \mathrm{CF}_{2} \mathrm{SO}_{3}$ the small value of $t_{z} \lesssim 0.2 \mathrm{~K}$ appears naturally because of the large interlayer distance $d \approx 17.5 \AA .22$.

\section{A. GaAs/AlGaAs heterostructures}

The area electron concentration $n_{e}=k_{F}^{2} / 2 \pi=6 \times$ $10^{11} \mathrm{~cm}^{-2}$ in the sample \#2 of GaAs/AlGaAs in Ref. [18] corresponds to the Fermi momentum $k_{F}=2 \times 10^{6} \mathrm{~cm}^{-1}$. This gives the cyclotron radius $R_{c}=\hbar k_{F} c / e B_{z}=$ $130 \mathrm{~nm} / B_{z}[T]$. Note that the magnetic length $l_{H}=$ $\sqrt{\hbar c / e B_{z}} \approx 2.5 \times 10^{-6} \mathrm{~cm} / \sqrt{B_{z}[T]}$ is of the same order as $R_{c}$, and the filling factor $\nu \sim 1$. The MQO frequency $F=k_{F}^{2} c \hbar / 2 e \approx 11.7 T$. The MQO and QHE are not observed because they are smeared completely by high temperature $T=75 \mathrm{~K}$. However, the localization of the electron states is preserved and affects the electron transport. Substituting $\varepsilon=12.4$ we obtain $E_{c} \approx 2 \pi e^{2} d / \varepsilon \pi R_{c}^{2} \approx$ $1.6 K \times B_{z}^{2}[T]$ and $X=\sqrt{4 E_{c} / T^{*}} \approx 0.3 B_{z}[T]$. Substituting this into Eq. (15) we obtain the dependence $R_{z z}\left(B_{z}\right)$. This dependence $R_{z z}\left(B_{z}\right)$ is shown in Fig. 2. where one only has to change the axis labels: on the ordinate axis $\alpha^{-1}(X) \rightarrow R_{z z}\left(B_{z}\right) / R_{z z}(0)$, and on the horizontal axis $X \rightarrow 0.3 B_{z}[T]$. The interval $0<B_{z}<13 T$ of magnetic field in Fig.1d of Ref. [18] corresponds to the interval $0<X<4$ in Fig. 2. This calculated dependence $R_{z z}\left(B_{z}\right)$ agrees very well with the experimental data plotted in Fig.1d of Ref. [18] at $\theta=0^{\circ}$, provided that there is a constant upward shift of the experimental curve $R_{z z}\left(B_{z}\right)$ in Ref. [18] which, probably, came from in-series resistances in the experimental setup. The crossover field, given by Eq. (10), $B_{c} \approx 0.5 T$ also agrees with the experimental data in Fig.1d of Ref. [18]. Unfortunately, there is no experimental data on the temperature dependence of the interlayer magnetoresistance in Ref. [18] to compare with out theoretical prediction.

\section{B. Organic metal $\beta$-(BEDT-TTF $)_{2} \mathbf{S F}_{5} \mathbf{C H}_{2} \mathbf{C F}_{2} \mathbf{S O}_{3}$}

The in-plane Fermi surface in the organic compound $\beta$-(BEDT-TTF) ${ }_{2} \mathrm{SF}_{5} \mathrm{CH}_{2} \mathrm{CF}_{2} \mathrm{SO}_{3}$ is an elongated ellipse with area [6] $\pi k_{F 1} k_{F 2}=0.0192 \AA^{-2}$, which is about $5 \%$ of the first Brillouin zone. The interlayer distance $d \approx 17.5 \AA$. Unfortunately, we are not aware of any measurements of the dielectric constant $\varepsilon$ in $\beta$-(BEDT$\mathrm{TTF})_{2} \mathrm{SF}_{5} \mathrm{CH}_{2} \mathrm{CF}_{2} \mathrm{SO}_{3}$. In organic metals the dielectric constant $\varepsilon$ varies in the interval from 8 to 50 and may depend on magnetic field, pressure and other external parameters. [47] Therefore, for the estimates below, we take the average value $\varepsilon \approx 20$. The exact value of the interlayer transfer integral $t_{z}$ in $\beta$-(BEDTTTF $)_{2} \mathrm{SF}_{5} \mathrm{CH}_{2} \mathrm{CF}_{2} \mathrm{SO}_{3}$ is not known yet, but the experimental estimates of the maximum value of $t_{z}$ give $t_{z} \leq 18.5 \mu \mathrm{eV}=0.21 \mathrm{~K}$. [22] Below we take $t_{z}=0.2 \mathrm{~K}$. The other parameters are $d=17.5 \AA$ and $F=200$ Tesla. Substituting this into Eqs. (10) or (11) we obtain the critical magnetic field $B_{c} \approx 8.5 T$ and the LL filling number $\nu_{c} \approx 23$ in a qualitative agreement with the experimental results in Refs. [21, 25]. Substituting the parameters to Eq. (9) we obtain $E_{c} \approx 0.012[K] \times B_{z}^{2}[T]$ and $X=\sqrt{4 E_{c} / T^{*}} \approx B_{z}[T] \sqrt{0.05[K] /\left(T+4 t_{z}\right)}$. Substituting this into Eq. (15) we obtain the dependence $R_{z z}\left(B_{z}, T\right)$ of the background magnetoresistance, averaged over MQO. This predicted dependence $R_{z z}\left(B_{z}\right) / R_{z z}(0)$ is shown in Fig. [3 for six different values of temperature: $T=0.66 \mathrm{~K}, 0.565 \mathrm{~K}, 0.455 \mathrm{~K}, 0.218$ $\mathrm{K}, 80 \mathrm{mK}$, and $47 \mathrm{mK}$. These values of temperature are taken to be the same as in Fig. 1 of Ref. 25], to simplify the comparison with experiment. As in Fig. 1 of Ref. [25], the highest curve in Fig. 3 corresponds to the lowest temperature. Comparison of Fig. 3 and Fig. 1 of Ref. 25] shows, that the agreement of the above theory with experiment is very good, both in the temperature and field dependence of magnetoresistance. 


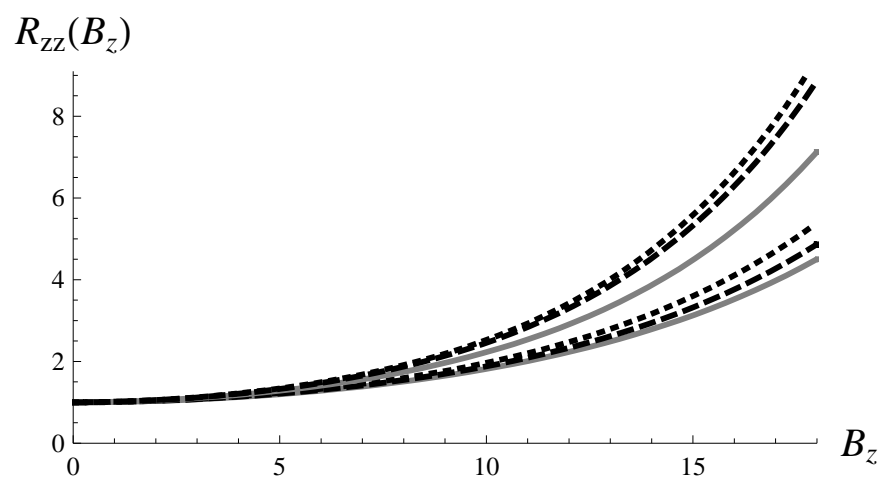

FIG. 3: The predicted field dependence of the background (non-oscillating) magnetoresistance $R_{z z}\left(B_{z}\right) / R_{z z}(0)$ in $\beta$ (BEDT-TTF $)_{2} \mathrm{SF}_{5} \mathrm{CH}_{2} \mathrm{CF}_{2} \mathrm{SO}_{3}$ at $t_{z}=0.2 \mathrm{~K}$ for six values of temperature: $T=0.66 \mathrm{~K}, 0.565 \mathrm{~K}, 0.455 \mathrm{~K}, 0.218 \mathrm{~K}, 80$ $\mathrm{mK}$, and $47 \mathrm{mK}$. The lower the temperature, the larger the magnetoresistance $R_{z z}\left(B_{z}\right)$, i.e. the highest curve in this plot corresponds to the lowest temperature. The values of temperature are taken to be the same as in Fig. 1 of Ref. [25].

\section{CONCLUSION}

Above we developed a theory to describe the interlayer magnetoresistance in the extremely anisotropic quasi-2D layered metals. Our model takes into account the po- laron effect. The polarons dress the localized electron states, which prevents the interlayer electron jumps by creating a Coulomb barrier. The energy of this Coulomb barrier, given by Eqs. (9) and (8), rapidly increases with the increase of the out-of-plane component $B_{z}$ of magnetic field because of the decrease of the electron localization length $\xi\left(B_{z}\right)$, given by Eqs. (4) and (5) [or by Eqs. (7) and (6) in the case of in-plane anisotropy]. The obtained temperature and field dependence of the interlayer conductivity is given by Eqs. (15)-(17). The comparison with experimental data on the field and temperature dependence of interlayer magnetoresistance in $\mathrm{GaAs} / \mathrm{AlGaAs}$ heterostructures and in organic metal $\beta$ (BEDT-TTF) ${ }_{2} \mathrm{SF}_{5} \mathrm{CH}_{2} \mathrm{CF}_{2} \mathrm{SO}_{3}$ is performed in Sec. IV. This comparison shows the nice qualitative and quantitative agreement of the proposed theory with experiments in very anisotropic layered metals even without any fitting parameters. It also explains long-standing problem of the strong field dependence of interlayer magnetoresistance in such compounds. The proposed theoretical model also allows to use the experiments on interlayer magnetoresistance to investigate the electron states, localized by magnetic field and disorder.

The work was supported by Russian Foundation for Basic Research, Foundation "Dynasty" and by LEA ENS-Landau exchange program.
[1] J. M. Ziman, Principles of the Theory of Solids, Cambridge Univ. Press 1972.

[2] A.A. Abrikosov, Fundamentals of the theory of metals, North-Holland, 1988.

[3] Shoenberg D. "Magnetic oscillations in metals", Cambridge University Press 1984.

[4] M.V. Kartsovnik, P.A. Kononovich, V.N. Lauk hin and I.F. Schegolev, JETP Lett. 48, 541 (1988) [Pis'ma Zh. Eksp. Teor. Fiz. 48, 498 (1988)].

[5] K. Yamaji, J. Phys. Soc. Jpn. 58, 1520 (1989).

[6] R. Yagi, Y. Iye, T. Osada and S. Kagoshima, J. Phys. Soc. Japan 59, 3069 (1990).

[7] M.V. Kartsovnik, P.D. Grigoriev, W. Biberacher, N.D. Kushch, P. Wyder, Phys. Rev. Lett. 89, 126802 (2002).

[8] P.D. Grigoriev, Phys. Rev. B 67, 144401 (2003).

[9] P.D. Grigoriev, M.V. Kartsovnik, W. Biberacher, N.D. Kushch, P. Wyder, Phys. Rev. B 65, 60403(R) (2002).

[10] M. V. Kartsovnuk and V. G. Peschansky, Low Temp. Phys. 31, 185 (2005) [Fiz. Nizk. Temp. 31, 249 (2005)].

[11] M.V. Kartsovnik, Chem. Rev. 104, 5737 (2004).

[12] T. Ishiguro, K. Yamaji and G. Saito, Organic Superconductors, 2nd Edition, Springer-Verlag, Berlin, 1998.

[13] J. Wosnitza, Fermi Surfaces of Low-Dimensional Organic Metals and Superconductors (Springer-Verlag, Berlin, 1996); J. Singleton, Rep. Prog. Phys. 63, 1111 (2000).

[14] N. E. Hussey, M. Abdel-Jawad, A. Carrington, A. P. Mackenzie and L. Balicas, Nature 425, 814 (2003).

[15] M. Abdel-Jawad, M. P. Kennett, L. Balicas, A. Carrington, A. P. Mackenzie, R. H. McKenzie \& N. E. Hussey, Nature Phys. 2, 821 (2006).
[16] M. Abdel-Jawad, J. G. Analytis, L. Balicas et al., Phys. Rev. Lett. 99, 107002 (2007).

[17] Malcolm P. Kennett and Ross H. McKenzie, Phys. Rev. B 76, 054515 (2007).

[18] M. Kuraguchi et al., Synth. Met. 133-134, 113 (2003).

[19] C. Bergemann, S. R. Julian, A. P. Mackenzie, S. NishiZaki, and Y. Maeno, Phys. Rev. Lett. 84, 2662 (2000); C. Bergemann, A. P. Mackenzie, S. R. Julian, D. Forsythe, and E. Ohmichi, Adv. Phys. 52, 639 (2003).

[20] P. D. Grigoriev, Phys. Rev. B 81, 205122 (2010).

[21] J. Wosnitza, S. Wanka, J. Hagel, H. v. Löhneysen, J. S. Qualls, J. S. Brooks, E. Balthes, J. A. Schlueter, U. Geiser, J. Mohtasham, R.W. Winter, and G. L. Gard, Phys. Rev. Lett. 86, 508 (2001).

[22] J.Wosnitza, J. Hagel, J. S. Qualls, J. S. Brooks, E. Balthes, D. Schweitzer, J. A. Schlueter, U. Geiser, J. Mohtasham, R. W. Winter, et al., Phys. Rev. B 65, 180506(R) (2002).

[23] M.V. Kartsovnik, D. Andres, S.V. Simonov, W. Biberacher, I. Sheikin, N. D. Kushch, and H. Müller, Phys. Rev. Lett. 96, 166601 (2006).

[24] M. V. Kartsovnik, P. D. Grigoriev, W. Biberacher, and N. D. Kushch, Phys. Rev. B 79, 165120 (2009).

[25] F. Zuo, X. Su, P. Zhang, J. S. Brooks, J. Wosnitza, J. A. Schlueter, Jack M. Williams, P. G. Nixon, R. W. Winter, and G. L. Gard, Phys. Rev. B 60, 6296 (1999).

[26] M. S. Nam, A. Ardavan, J. A. Symington, J. Singleton, N. Harrison, C. H. Mielke, J. A. Schlueter, R. W. Winter, and G. L. Gard, Phys. Rev. Lett. 87, 117001 (2001).

[27] T. Champel and V. P. Mineev, Phys. Rev. B 66, 195111 
(2002).

[28] P. Moses and R.H. McKenzie, Phys. Rev. B 60, 7998 (1999).

[29] P.D. Grigoriev, Phys. Rev. B 83, 245129 (2011).

[30] P. D. Grigoriev, JETP Lett. 94, 47 (2011).

[31] P. D. Grigoriev, Fizika Nizkikh Temperatur (Low Temperature Physics) 37, 930 (2011).

[32] Tsunea Ando, J. Phys. Soc. Jpn. 36, 1521 (1974).

[33] W. Kang, Y. J. Jo, D. Y. Noh, K. I. Son, and Ok-Hee Chung, Phys. Rev. B 80, 155102 (2009).

[34] V. M. Gvozdikov, Phys. Rev. B 76, 235125 (2007).

[35] M. M. Fogler, A. Yu. Dobin, V. I. Perel, and B. I. Shklovskii, Phys. Rev. B 56, 6823 (1997).

[36] M. M. Fogler, A. Yu. Dobin, and B. I. Shklovskii, Phys. Rev. B 57, 4614 (1998).

[37] M. E. Raikh and T. V. Shahbazyan, Phys. Rev. B 51, 9682 (1995).

[38] B. Huckestein, Rev. Mod. Phys. 67, 357 (1995).

[39] M. Amado, A. V. Malyshev, A. Sedrakyan, and F. Dominguez-Adame, Phys. Rev. Lett. 107, 066402 (2011).

[40] M. Furlan, Phys. Rev. B 57, 14818 (1998).

[41] R. M. Lewis and J. P. Carini, Phys. Rev. B 64, 073310 (2001).

[42] F. Hohls, U. Zeitler, and R. J. Haug, Phys. Rev. Lett. 86, 5124 (2001).

[43] F. Hohls, U. Zeitler, and R. J. Haug, Phys. Rev. Lett. 88, 036802 (2002).

[44] The exact theoretical calculation of the localization length and its dependence on the LL filling factor $\nu$ is rather complicated, and the attempts to improve the accuracy of the estimates of $\xi$ continue nowadays. 35 39] At integer LL filling factor $\nu$, i.e. when the chemical potential is between the LLs, both the calculations 35 37) and the experimental estimates 40 42 give $\xi \approx R_{c}$. At half-integer $\nu$ the theory predicts a divergence 38, 39] $\xi(\nu) \propto|\Delta \nu|^{-\gamma}$ with $\gamma \approx 2.4 \pm 0.2$. The exeriments, performed only at low filling factor $\nu$, confirm that at low temperature and in clean materials the function $\xi(\nu)$ may increase by an order of magnitude when $\nu$ changes from integer to the half-integer values. [40 43] The disorder, finite temperature and interlayer transfer integral smear this sharp dependence $\xi(\nu)$, especially at $\nu \gg 1$. The experimental determination of the localization length in Refs. [38, 40 43] is based on the in-plane electron transport in the variable-range hopping regime. This means that an exponentially small amount of delocalized electron states at half-integer $\nu$ may give the main contribution to $\sigma_{x x}$ and to the divergence of $\xi$, while the major part of electron states remain localized on the length $\xi \approx R_{c}$. Contrary to the in-plane conductivity $\sigma_{x x}$, the interlayer electron transport is almost not affected by this extremely small amount of delocalized states. Therefore, for the estimates of interlayer magnetoresistance below we take $\xi \approx R_{c}$ in Eqs. 4 and 7 .

[45] E. Chashechkina \& P. Chaikin, Phys. Rev. Lett. 80, 2181 (1998).

[46] J. S. Brooks, V. Williams, E. Choi, D. Graf, M. Tokumoto, S. Uji, F. Zuo, J. Wosnitza, J. A. Schlueter, H. Davis, R. W. Winter, G. L. Gard and K. Storr, New J. Phys. 8255 (2006); [doi:10.1088/1367-2630/8/10/255].

[47] M.Dressel, G.Grüner, J.P.Pouget, A.Breining, D.Schweitzer, J.Phys. I France 4, 579 (1994). 